\title{
Study on Diesel Engine Status Analysis and Fault Diagnosis Based on SAE J1939 Protocol
}

\author{
L.H. Cao, J.N. Li, X.L. Liu, J.L. Yu, Z.M. Wu, F.G. Chen \\ Control Engineering Key Laboratory \\ Chongqing Communication Institute \\ Chongqing,China
}

\begin{abstract}
SAE J1939 is one of the most widely used application layer protocols in automotive industry based on CAN bus. SAE J1939 defines vehicle application layer to make us read the status of the vehicle easily. We propose an improved RBF neural network fault classification algorithm to classify diesel engine fault. Also we analyse the state of the vehicle from vehicle CAN bus and use MATLAB to write a program so that we can analyse the CAN bus data. According to CAN data obtained from a diesel engine, we got diesel engine fault data and use they to train PSO-RBF neural network, the resulting effect is better than PSO neural network and we can use it to do a diesel engine fault diagnosis.
\end{abstract}

Keywords-SAE J1939; diesel engine; fault diagnosis; PSO-RBF neural network

\section{INTRODUCTION}

With the development of the automobile industry and electronic control technology, getting data of vehicle fault is no longer only rely on traditional method. Traditional methods such as extracting eigenvalues of the vibration signal, hydraulic waveforms to diagnosis engine fault. Due to it is analyzed by one data source, it has a high probability of misdiagnosis. Therefore, vehicle fault diagnosis based on a variety of data sources has become a research focus.

SAE J1939 developed by the American Society of Automotive Engineers, is the vehicle bus recommended practice used for communication and diagnostics among vehicle components, originally by the car and heavy-duty truck industry in the United States [1]. SAE J1939 defines four layers in the seven-layer OSI network model: physical layer, data link layer, network layer, vehicle application layer, and the physical layer is based on CAN controller [2]. SAE J1939-71 developed for the application layer is to make the data provided by source node can be used by the other nodes [3].

Engine fault diagnosis is also largely dependent on human experience and traditional diagnostic methods currently, fault diagnosis based on SAE J1939 protocol is still in the development stage. We import the data which obtained in the operation of the vehicle into MATLAB. Analysis in MATLAB is effective that we can analyze the state of vehicle and diagnose whether the vehicle has a potential fault.

\section{SAE J1939 PROTOCOL}

Open System Interconnection Reference Model (OSI/RM) is a networking model proposed by International Organization for Standardization (IOS). OSI protocols stacks are split into seven layers. CAN protocol only defines physical layer and data link layer. SAE J1939 protocol is based on CAN2.0B, and defined network layer and application layer in addition.

\section{A. Data Link Layer}

Data link layer provides reliable data transmission between physical connections, includes sending synchronization, sequence control, error control and flow control which is necessary for CAN data frame [4]. In the CAN standard, MAC ID has two standards: CAN2.0A, which is 11-bit, and CAN2.0B, which is 29-bit. The structure difference is mainly in arbitration field and control field [5]. J1939 uses CAN2.0B communication mechanism. The physical layer follows electrical characteristics, communication media, physical interface of CAN protocol. In data link layer, J1939 redefines 29-bit ID of arbitration field and 64-bit data of data field corresponding to vehicle information [6].

Protocol data unit consists of seven parts: priority, extended data page, data page, PDU format, PDU specific domain, source address and data fields. PDU is encapsulated in one or more CAN data frames to transmit to the other network devices through physical medium. Each CAN data frame has only one PDU. The PDU format is as Table 1.

TABLE I. PDU FormAT OF SAE J1939 PROTOCOL

\begin{tabular}{|c|c|c|c|c|c|c|c|}
\hline $\begin{array}{c}\text { Nam } \\
\text { e }\end{array}$ & $\begin{array}{c}\text { Priorit } \\
\mathbf{y}\end{array}$ & $\begin{array}{c}\text { Extende } \\
\text { d data } \\
\text { page }\end{array}$ & $\begin{array}{c}\text { Dat } \\
\text { a } \\
\text { pag } \\
\text { e }\end{array}$ & $\begin{array}{c}\text { PDU } \\
\text { forma } \\
t\end{array}$ & $\begin{array}{c}\text { PDU } \\
\text { specifi } \\
\text { c } \\
\text { domai } \\
\text { n } \\
\end{array}$ & $\begin{array}{c}\text { Source } \\
\text { addres } \\
\quad \mathrm{S}\end{array}$ & $\begin{array}{l}\text { Data } \\
\text { fields }\end{array}$ \\
\hline $\begin{array}{c}\text { Abbr } \\
\text {. }\end{array}$ & $\mathrm{P}$ & EDP & DP & $\mathrm{PF}$ & PS & SA & $\begin{array}{l}\text { DAT } \\
\text { A }\end{array}$ \\
\hline bit & 3 & 1 & 1 & 8 & 8 & 8 & 64 \\
\hline
\end{tabular}

\section{B. Application Layer}

Application layer of J1939 is stipulated by J1939-71. It provides a way to access the OSI environment and describes packet format, data range, transmission repetition rate, parameters naming and ISO Latin character through PGN (Parameter Group Number) and SPN (Special Parameter Number). 
A set of parameters called "parameter group" (PG), PGN is its unique identification number. PGN serves as an index in the communication process. It shows the contents and purpose of the message, determines packet type and number of frames. Here is an example, PGN 61444: Default Priority is 011, Extended Data Page is 0, Data Page is 0, PDU Format is 11110000, PDU Specific is 00000100 and Data fields as follow:

TABLE II. SPNS OF PGN 61444

\begin{tabular}{clll}
\hline $\begin{array}{c}\text { Start } \\
\text { Position }\end{array}$ & Length & \multicolumn{1}{c}{ Parameter Name } & SPN \\
\hline $\mathbf{1 . 1}$ & 4bit & $\begin{array}{l}\text { Engine Torque Mode } \\
\text { Driver's Demand Engine }\end{array}$ & 899 \\
$\mathbf{2}$ & 1byte & $\begin{array}{l}\text { Percent Torque } \\
\text { Actual Engine - Percent }\end{array}$ & 513 \\
$\mathbf{3}$ & 1byte & $\begin{array}{l}\text { Torque } \\
\text { Engine Speed }\end{array}$ & 190 \\
$\mathbf{4 - 5}$ & 2byte & $\begin{array}{l}\text { Source Address of } \\
\text { Controlling Device for }\end{array}$ & 1483 \\
$\mathbf{6}$ & 1byte & $\begin{array}{l}\text { Engine Control } \\
\text { Engine Starter Mode } \\
\text { Engine Demand - Percent } \\
\text { Torque }\end{array}$ & 1675 \\
$\mathbf{8}$ & 4bit & 1byte & 2432 \\
\hline
\end{tabular}

Parameters of PGN 61444 represent electronic engine controller 1, which composed of seven specific parameters. For example in SPN190, SPN 190: data length is 4 bits, resolution is 16 states/4 bit and 0 offset, data range is 0 to 15 , operational range is also 0 to 15 , type is Measured. If we got the data of SPN 190 is 201B, it shows that the engine speed is $868 \mathrm{rpm}$. The other data can be got as the same way.

We can get a variety of vehicle status data based on SAE J1939 protocol. It is convenient for us to have a comprehensive understanding of the performance of vehicle. But the data is too scattered and inconvenient to understand, we need an analytical tool to analyze the data.

\section{DATA ANALYSIS OF SAE J1939-71 IN MATLAB}

The most important in J1939 protocol is PGN and SPN. We create two new cell type: PGN \{\}$, \operatorname{SPN}\{\}$. PGN \{\} include: Name, Transmission Repetition Rate, Data Length, Extended Data Page, Data Page, PDUF or mat, PDU Specific, Default Priority, SPNNUM, SPN \{\} . SPN \{\} include: Name, Data Length, Resolution, Data Range, Type.

After acquiring CAN data, we import the data into MATLAB as cell type. Flow diagram of J1939 data conversion is as Figure 1.

$\operatorname{SPN}\{\}$.Out M() is the output of the SPN at the moment.

We get Diesel Exhaust Fluid injection unit input pipe failure data of Cummins 6B diesel engine, imported into MATLAB for analysis. Take SPN(190) and SPN(110) for example, the output shown in Figure 2. It shows the situation within this period of time the engine speed and the engine coolant temperature.

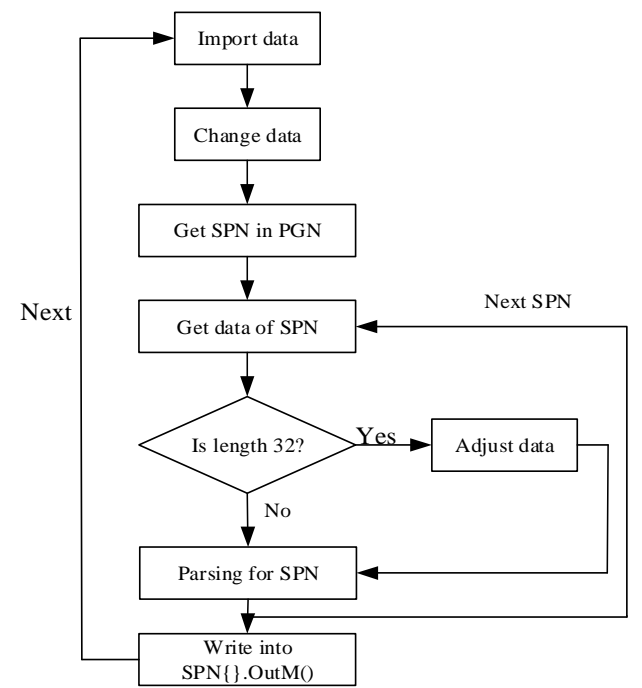

FIGURE I. FLOW DIAGRAM OF J1939 DATA CONVERSION

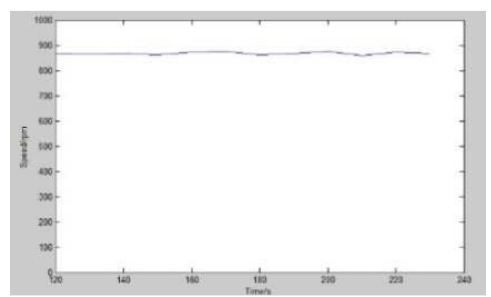

(1) $\operatorname{SPN}(190)$

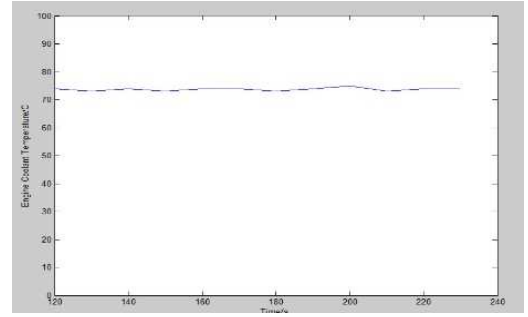

(2) $\operatorname{SPN}(110)$

FIGURE II. SPN OUTPUT

After the analysis of the parameters we need, we can have an overall grasp of the vehicle's condition. When we analyze engine failure, we can choose targeted fault parameters forming fault parameter table, then use different fault diagnosis algorithm to do engine fault diagnosis.

\section{DiesEl ENGine FAUlt Diagnosis BASED ON IMPROVED RBF NEURAL NETWORK}

\section{A. RBF Neural Network}

RBF neural network is two forward lays network with a single hidden layer. The input layer consists of source node. The number of hidden layer units depend on the fixed problem. Transformation from the input space to the hidden layer space is nonlinear, and from the hidden layer to the output layer space transform is linear. Hidden layer node consist of functions like Gaussian function. Output layer node is typically a simple linear function. The topology structure shown in Figure 3. 


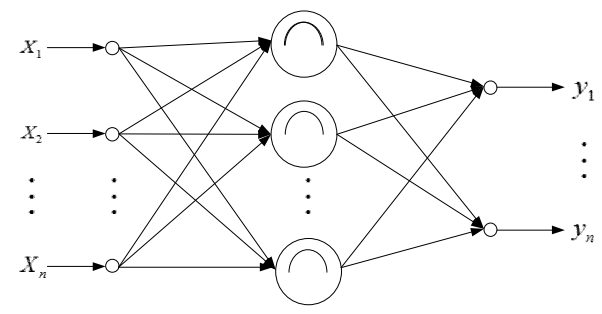

FIGURE III. THE TOPOLOGY STRUCTURE DIAGRAM OF RBF NEURAL NETWORK

Transformation function for hidden node is radial basis function. It takes Gaussian function normally:

$$
\Phi_{j}=\exp \left[-\frac{\left\|x-c_{j}\right\|}{2 \delta_{j}^{2}}\right] \quad j=1,2, \cdots, q
$$

$i_{\text {-th output of the network is: }}$

$$
y_{i}=\sum_{j=i}^{q} \omega_{j i} \Phi\left(x, c_{j}, \delta_{j}\right)_{i=1,2, \cdots, m}
$$

$x$ is the input vector, ${ }^{c_{j}}$ is center of the hidden layer unit, $\delta_{j}$ is width, $\omega_{j, i}$ is the connection weights. Because the value of $c^{c_{j}}, \delta_{j}, \omega_{j, i}$ has a great influence on the learning and training effectiveness, for how to determine $^{c_{j}}, \delta_{j}, \omega_{j, i}$, we use PSO to optimize these three parameters.

Particle swarm optimization (PSO) algorithm deemed individuals to particles in $\mathrm{N}$-dimensional search space. Each particle follow current best individual and change their speed and location through sharing information and find the optimal solution through continuous iteration eventually. The algorithm first randomly generated particle swarm, each particle has a speed and position in the N-dimensional solution space. Speed and position are adjusting by their own condition and the experience of other particles. Finally, reaching the maximum number of iterations or PSO searched the optimal fitness value [7].

PSO-RBF algorithm is as follows:

Each particle consist of ${ }^{c_{j}}, \delta_{j}, \omega_{j, i}$, random initial its speed and position.

$$
\text { Set } f=\frac{1}{N} \sum_{i=1}^{m}\left|\frac{y_{i}^{\prime}-y_{i}}{y_{i}}\right| \text { as a fitness function to determine }
$$
the best individual particles $p_{\text {best }}$ and global optimization $^{g_{\text {best }}}$. ${ }^{\prime}{ }_{i}^{\prime}$ is the actual value and $y_{i}$ is output value.

Compare the fitness value of each particle with best individual particles and global optimum. If $f\left(P_{i}\right)>f\left(p_{\text {best }}\right)$, then $p_{\text {best }}=P_{i}$. If $f\left(P_{i}\right)>f\left(g_{\text {best }}\right)$, then ${ }^{g_{\text {best }}}=P_{i}$.
Each particle update their speed and position.

If it does not met the termination condition, then return (2), otherwise, stop.

\section{B. Engine Fault Diagnosis Based on Improved RBF Neural Network}

We got 84 sets of data of seven kinds of faults which are got from CAN bus of Cummins $6 \mathrm{~B}$ diesel engine in a truck( A:Diesel Exhaust Fluid injection input unit has problem, B: fuel rail pressure of injector is below the normal operating range, C:Diesel Exhaust Fluid reservoir level is below the normal operating range, D:power losing when the ignition switch is turned on, E:engine control module calibration memory damages, F:speed is unstable, intermittent or error which based on the number of revolutions of the wheel, G:sensor of nitrogen oxides exporting updates rate abnormally). After MATLAB analysis, some of the data as follows:

TABLE III. FAUlts Data of DiEsel Engine

\begin{tabular}{lllllllll}
\hline No. & $\mathbf{X 1}$ & $\mathbf{X 2}$ & $\mathbf{X 3}$ & $\mathbf{X 4}$ & $\mathbf{X 5}$ & $\mathbf{X 6}$ & $\mathbf{X 7}$ & $\begin{array}{c}\text { Fault } \\
\text { Code }\end{array}$ \\
\hline $\mathbf{1}$ & 1079 & 23 & 249.3 & 74 & 868 & 117 & 130 & 1 \\
$\mathbf{2}$ & 85 & 0 & 0.5 & 30.3 & 191 & 30 & 30 & 2 \\
$\mathbf{3}$ & 915 & 0 & 0 & 77 & 0 & 0 & 68 & 3 \\
$\mathbf{4}$ & 723 & 0 & 0 & 17.2 & 0 & 0 & 11 & 4 \\
$\mathbf{5}$ & 906 & 0 & 173 & 76.9 & 701 & 100 & 100 & 5 \\
$\mathbf{6}$ & 1125 & 0 & 338 & 58.1 & 861 & 135 & 114 & 6 \\
$\mathbf{7}$ & 1275 & 16 & 503 & 45.5 & 1684 & 15 & 61 & 7 \\
$\ldots$ & $\ldots$ & $\ldots$ & $\ldots$ & $\ldots$ & $\ldots$ & $\ldots$ & $\ldots$ & $\ldots$ \\
$\mathbf{8 4}$ & 1312 & 15 & 506 & 45.8 & 1669 & 14 & 60 & 7 \\
\hline
\end{tabular}

$\mathrm{X} 1$ is measured fuel rail pressure, $\mathrm{X} 2$ is speed, $\mathrm{X} 3$ is oil pressure, $\mathrm{X} 4$ is coolant temperature, $\mathrm{X} 5$ is rotational speed, $\mathrm{X} 6$ is SCR outlet temperature and X7 is SCR inlet temperature.

Select 56 groups as the training sample, and the rest as a test sample. Use training samples to train PSO-RBF neural network, the 7 input vector are $\mathrm{X} 1$ to $\mathrm{X} 7$. There are 7 kinds of faults, replaced by code 1 to 7 . One hidden layer and containing 25 hidden layer units. PSO parameters are set as follows: scale is 500 , inertia weight factor is 0.9 , learning factor $c_{1}=c_{2}=2$, termination condition is that error is less than 0.01 or reaching 100 generations. PSO-RBF and RBF are used for training. The convergence curve lines is shown in Figure 4.

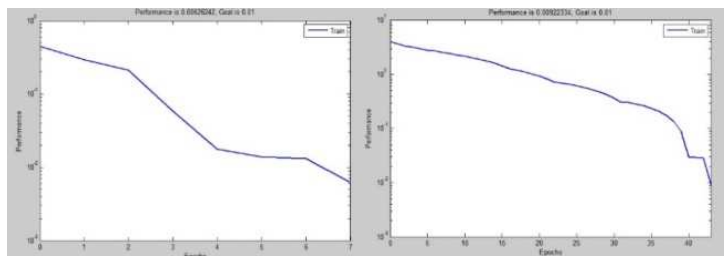

(1) PSO-RBF

(2) RBF

FIGURE IV. THE CONVERGENCE CURVE LINES OF PSO-RBF AND RBF ALGORITHMS

Convergence rate of PSO-RBF is faster. We use the trained network to classify the remaining 28 groups, the results are as follows. It shows that PSO-RBF has higher accuracy and 
shorter training time.

TABLE IV. THE DiAgNostic RESULtS AND TRAINING TIME

\begin{tabular}{cll}
\hline & \multicolumn{1}{c}{ PSO-RBF } & \multicolumn{1}{c}{ RBF } \\
\hline Accuracy & $89.3 \%$ & $78 \%$ \\
Time & $1.636 \mathrm{~s}$ & $5.785 \mathrm{~s}$ \\
\hline
\end{tabular}

\section{V.SUMMARY}

Rely on powerful data processing capability and convenient language compiler environment of MATLAB, data processing base on $\mathrm{J} 1939$ has a unique position. We are able to process and analyze a variety of data to have comprehensive and accurate understanding of information on the vehicle. This can be an efficient analysis tool for vehicle detection and repairing. After analyze the data, we use PSO-RBF neural network algorithm to diagnose vehicle fault with a high accuracy. The algorithm can be improved in the future to make the accuracy to $100 \%$ and a shorter time.

\section{ACKNOWLEDGEMENTS}

Supported by the Outstanding Achievements Transformation Funded Major Projects of Chongqing College (Grant No. KJZH14112).

\section{REFERENCES}

[1] SAE J1939 Standards Collection, Recommended Practice for a Serial Control and Communication Vehicle Network[S]. Society of Automotive Engineers, 2003.

[2] Wu Jianfang. Research of Embedded Vehicle Information Terminal Implementation [D]. Harbin: Harbin Engineering University, 2010.

[3] SAEJ1939-71 Vehicle Application Layer[S]. USA. SAE. 2006.

[4] Hu Yajuan. Design and Implementation of Vehicle Information Display System Based on J1939 Protocol [D]. Chengdu: Southwest Jiaotong University, 2008.

[5] Zeng Hongan. Embedded Linux C Programming [M]. Beijing: Publishing House of Electronic Industry.

[6] Zhang Qianqin. Research on the Control System about Central Signals in Electric Vehicle [D]. Beijing: Beijing Jiaotong University, 2010.

[7] Kennedy J, Eberhart R.C., Particle swarm optimization [J]. Proceedings of IEEE International Conference on Neural Networks. 1995. 1942-1948. 